lich mit der quantität der wurzelsilbe zusammen, noch wurde secundär eine derartige verteilung je durchgefuhrt. Die verschiedene behandlung der kurz- und langsilbigen io-stämme geht entweder direct oder doch wenigst indirect auf lautliche processe zurück.

HEIDELBERG, november 1889.

M. H. JELLINEK.

\title{
GERMANISCH $\hat{E}^{2}$.
}

Mahlow, Die langen vocale AE0 s. 163 erklärt westgerm. hêr als contraction aus *hear, *hiar und meint, dass ein pronominalstamm *hja wie in hiutagu, heódcez vorliege, resp. sei die form durch anfügung der endung der $a$-stämme aus der form der $i$-stämme entstanden. Möglicherweise sei auch got. her aus *hear hervorgegangen, indem ein vorauszusetzendes * herr von har, par die endung -ar übernahm.

Diese theorie ist etwas weiter ausgefubrt worden von O. Schrader, der BB. 15, 131 die beobachtung zu machen glaubt, dass germ. $\hat{e}^{2}$ immer vor $r$ stehe, und den laut als urgerm. contractionsproduct von -ija- vor $r$ erklärt. Ich halte diese ansicht, der sich neuerdings auch Kluge, Pauls Grundr. I, 356 anschliesst, fur ganz verfehlt. Erstlich steht $\hat{e}^{2}$ nicht nur vor $r$, was weiter unten geezeigt werden soll. Danu ist auch Schraders ausgangspunct unglticklich gewählt. Weil hêr einerseits nicht von dem pronominalstamm $h i$, andererseits nicht von par, war etc. getrennt werden könne, habe es den anschein, als ob hêr aus hiiar contrahiert sei. Ob der verweis auf Mahlow besagen soll, dass Schrader sich ganz der argumentation dieses gelehrten anschliesst, ist nicht ersichtlich. Aber nur wenn man Mahlows theorie von einem stamm hja acceptiert, kann man von einem germ. par auf ein germ. hiinar schliessen, doch fehlen fur die existenz eines solchen stammes jegliche anzeichen. Auf heodaez darf man sich nicht berufen, denn der instrumental der $i$ - und der $i 0$-stämme war im idg. gleichlautend. Auch, dass die formen par, war die entstehung eines germ. *hiiar bewirkt hätten, ist unglaublich. Ueberall 
dort wo die germ. ståmme $p a, h w a$ den stammauslaut $a$ aufweisen, zeigt der stamm $h i$ ein $i$. In der flexion: himma, hina, hita wie pamma, pana pata, vor adverbialsuffixen: got. hidre wie hadre, ahd. hina wie dana, hëra wie dara. Es ist nach Mahlow-Schraders theorie nicht einzusehen, warum es nicht auch *hêra heisst. Die dem par entsprechende form vom stamm $h i$ ist $h i{ }^{1}$ ) und liegt auch tatsächlich im alts. vor. Es ist auch durchaus nicht von vorneherein selbstverständlich, dass man hêr gerade mit formen wie par in beziehung setzén muss. In ausschliesslichem gebrauch sind die ruheadverbia auf -ar nur im got. und altn.; in den wg. sprachen sind die regulären formen solche wie ags. pér, ahd. $d a ̂ r$, für die man eine grundform. germ. * pêr ansetzen muss. Wenn wir nun finden, dass im germ. von pronominalen $a$-stämmen zwei formen der ruheadverbia vorhanden sind, eine auf -ar und eine auf -êr, ebenso vom stamme hi zwei formen' hir und hêr; von denen hir dem par entspricht, so scheint es geboten hêr mit ags. pér zusammenzustellen.

Die Schradersche hypothese scheitert endlich auch daran, dass die lautgruppe iiar unversehrt erhalten ist in got. un-

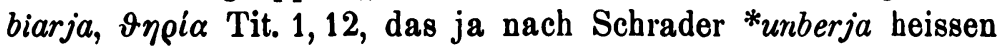
musste.

Soviel ergibt sich aber aus den bekannten zusammenstellungen der $\hat{e}^{2}$-wörter mit etymologisch verwanten, zu denen Schrader in seinem aufsatz durch heranziehung der anssergermanischen sprachen dankenswerte erganzungen geliefert hat, dass neben den formen mit $\hat{e}$ solche mit $\vec{\imath}$ stehen. Es scheint also, dass man germ. $\hat{e}^{2}$ als einen ablaut der $e i-r e i h e$ zu betrachten hat.

Das beweisen auch die folgenden zusammenstellungen, die sich auf wörter beziehen, in denen $\hat{e}^{2}$ nicht vor $r$ steht.

Ags. cén, ahd. kên, kien gehört wol zu keinan, ahd. siiega sicher zu stigan. Dass hier ursprüngliches $\hat{e}$ vorliegt, beweisen die Mons. gl. die ie nur fur $\hat{e}^{2}$ kennen ${ }^{2}$ ). Neben ahd.chreg,

1) Das hat schon J. Schmidt, KZ. XXVII, 303 ausgesprochen, obwol ihm das tatsächliche vorkommen von hir im alts. entgangen war, 8. w. unteu.

2) strez Ahd. gl. I, 633, 66. 657, 10, stiezun 662, 88. 753, 27 sind angleichungen an die praeterita der übrigen ehemals reduplicierenden verba. 
mhd. kriec steht nl. krijg, im mhd. finden sich die verba kriegen und krigen nebeneinander. $i e$ neben $i$ steht ferner in mhd. Kriemhilt neben Krimhilt, altn. Grimhildr, mhd. Vriesen neben lat. Frisiones, ags. Frisan, Fresan. Vgl. auch schief, wenn es zu altn. skeifr etc. gehört.

Germ. $\hat{e}^{2}$ liegt auch in dem namen Wieland, ags. Wéland vor $\left.{ }^{1}\right)$. Ich möchte ihn mit der wurzel ui 'drehen, winden' in beziehung setzen. Ein von dieser wurzel abgeleitetes uilo-, neben dem gleichberechtigt $u \bar{e}^{2} l o-$ stand, kann die bedeutung 'das gedrehte, gewundene' gehabt haben, vgl. lat. templum urspr. das abgeschnittene. Wéland wäre dann das ptep. praes. eines von diesem $u \bar{e}^{2} l o-$ abgeleiteten schw. verbs. Wéland hiesse also ursprünglich 'der dreher'. Ags. wir, ahd. wiara, in dem dieselbe wurzel $u i$ steckt hat die bedeutung 'gewundener schmuck'. Aehnlich wird Wéland prägnant 'verfertiger von gewundenem schmuck, von ringen' bedeutet haben. Ein appellativ dieser bedeutung eignete sich ganz gut zur bezeichnung des kunstfertigen schmiedes. Dass handwerker nach einem einzelnen producte ibrer tätigkeit benannt werden, ist eine bekannte tatsache; dabei können verschiedene erzeugnisse als besonders charakteristisch erscheinen. Vgl. schreiner und tischler als benennungen desselben handwerkers.

Schwierigkeit macht das verhältnis von got. mizdo zu den entsprechenden formen der verwanten dialekte. Dass alts. mêda etc. die directe fortsetzung der got. form ist, scheint wenig glaublich, da im ags. neben dem gewöhnlichen méd auch meord vorkommt. Vielleicht liegt auch hier ein ablaut vor und $z$ ist wg. nach $\hat{e}^{2}$ ausgefallen. Mhd. wiege scheint freilich zu der in wëgan vorliegenden $e$-wurzel zu gehören. Aber schon die ahd. form wiga macht schwierigkeiten, da man *wëga oder * wicka erwarten sollte. Vielleicht sind zwei ursprunglich verschiedene wurzeln germ. weg und wig anzunehmen.

Es fragt sich nun welche stelle nahm $\hat{e}^{2}$ in der ei-reihe ein? Aus der vergleichung von ags. hér und bér, d. i. germ. $h \bar{e}^{2} r$ und $p \bar{e}^{1} r$, scheint sich zu ergeben, dass einem $\bar{e}^{1} \mathrm{der}$

1) Ueber das verhältnis dieser zu der nord. form Velundr vgl. Symons Pauls Grdr. II, 61. 
$e / 0$-reihe ein $\bar{e}^{2}$ der $e i$-reihe entspricht. Daraus scheint weiter zu folgen, dass $\bar{e}^{2}$ in germ. aus $\bar{e} i$ entstanden ist.

Doch erheben sich gegen diese annahme bedenken. Zwar Bremers theorie, dass $\bar{e} i$ im germ. auch in haupttoniger silbe zu ai geworden sei (Beitr. XI, 41), darf durch die ausfuhrungen Osthoffs, Beitr. XIII, 444 ff. als beseitigt angesehen werden. Gewichtiger sind die einwände, die man aus der von J. Schmidt aufgestellten theorie von dem idg. ausfall des $i$ nach langen vocalen vor consonanten schöpfen könnte. Ich habe trotzdem meine hypothese von der entstehung des $\bar{e}^{2}$ nicht verschweigen wollen, weil sie vom germ. standpuncte aus die wahrscheinlichste ist und Schmidts theorie, soweit ich sehe, noch nicht allgemein acceptiert ist. Dann scheint mir auch sehr beachtenswert, was Brugmann, Grundr. II. $\S 160$ a. 3 bemerkt, dass nämlich nicht alle wörter dieser art zu gleicher zeit aufgekommen sein mussen. Bei *hēr lässt sich im einzelnen zeigen, wie die richtigkeit der Schmidtschen theorie zugegeben doch ein antecons. $\bar{e} i$ möglich war.

Schmidt vermutet KZ. XVII, 303, dass die lautgesetzliche form hir durch einwirkung des loc. ${ }^{*} h \bar{e}$ zu her geworden sei. Dabei bleibt aber die qualität des $\bar{e}$ unerklärt. Auch wird nicht berlicksichtigt, dass vom stamm $p a$ zwei formen des adv. der ruhe vorliegen, ein gleiches also vom stamme hi zu erwarten ist. Endlich scheint mir eine contamination, wie sie Schmidt voraussetzt, wenn auch natlirlich nicht unmöglich, so doch weniger einleuchtend als die analogiewirkung, die ich im folgenden auseinandersetzen will.

Einem *ber (ags. pár) entsprach ein ursprungliches *heir. Daraus wurde schon vorgerm. nach Schmidts gesetz *hèr mit einem $\bar{e}$, dessen qualität sich in nichts von dem in *be r unterschied. Der loc. des stammes $h i$ lautete $* h \bar{e}$. Daneben bildete sich eine form *heie, wie ähnliches Schmidt ja auch sonst annimmt. (Vgl. a.a. 0. 303, wo anstai aus *anstēi erklärt wird.1)) Nach dem verhältnis *hē :hēr bildete sich durch einwirkung

1) Oder es bestand schon im idg. eine satzdoppelform *hëi, vgl. Bremer, Beitr. XI, 37 f., Meringer, Zs. fög. 39, 132 ff. J. Schmidt, Festgruss an Böhtlingk, s. 102. 
ron *hēi die form *heir. Auf germ. boden wurde dann *hēir bei starker betonung zu $h \bar{e}^{2} r$. (In enklitischer stellung wäre *hair entstanden.)

M. H. JELLINEK.

\section{ZUM HELIAND.}

I. So oft rom dialekt des Mon. die rede ist, wird Heynes hypothese (Zs. fdph. I, 288) von dem munsterländischen ursprung der handschrift, erwähnt, freilich mehr, weil man nichts besseres an ibre stelle setzen kann, als weil sie besonderen anklang gefunden bätte. Das argument Heynes ist die angeblich besonders nahe sprachliche verwantschaft des Mon. und der Freckenhorster heberolle. Was davon zu halten ist, sollen die folgenden ausfuhrungen lehren.

Eine vergleichung von Mon. und Freck. ist nicht obne schwierigkeiten, denn die heberolle ist vollständig nur in einer hs. des ausgehenden 11. jh. Uberliefert ${ }^{1}$ ) und vom Mon. kann es jetzt als ausgemacht gelten, dass die dialekte der vorlage und des schreibers nicht identisch waren. Nur um die frage kann es sich handeln, ob der schreiber von Mon. ein Munsterländer war oder nicht, denn die vorlage weicht in drei charakteristischen puncten von Freck. ab: sie gebrauchte im d. sg. m. n. der adj. die kurzere form auf -on, der a. sg. m. des dem. lautete thana, die präp. von fon ${ }^{2}$ ) Freck. bietet dagegen ebenso wie der schreiber von Mou. dative auf -omo, -amo, -emu, thena ist am häufigsten belegt (6 mal, then 2 mal, than 1 mal) und die präp. lautet ausnahmlos van.

Sonst sind folgendes die charakteristischen eigenheiten der heberolle:

\section{Vocalismus der stammsilben.}

1. $o$ hat die neigung in $a$ uberzugehen: z. b. hanigas 9 . 191. 224. 229 in $\mathrm{K}$ der ältern hs. neben 1 mal belegtem honi-

1) Beyne, Kl. altnd. Denkm. s. 67. Ich citiere immer nach dieser ausgabe.

2) Vgl. Beitr. XII, 287. XIV, 158, Germ. 31, 380. 